\title{
Reviewer acknowledgement 2014
}

Philippa K Harris

\section{Contributing reviewers}

The editors of BMC Developmental Biology would like to thank all our reviewers who have contributed to the journal in Volume 14 (2014).

\author{
Craig Albertson \\ USA
}

Declan W. Ali

Canada

Jeffrey Amack

USA

Ana Angelova Volponi

UK

Christopher Antos

Germany

Michalis Averof

France

Alfred Batschauer

Germany

Nathalie Beaujean

France

Mark Berryman

USA

Marco Biggiogera

Italy

Samuel Breit

Australia

Cathrin Brisken

Switzerland

Aaron Brown
USA

Thomas Carroll

USA

\author{
Francesc Cebria \\ Spain \\ Sang-Wook Cha \\ USA \\ Tatiana Chebotareva \\ UK \\ Steven Cheng \\ China
}

Rosanna Chianese

Italy

Ariel Chipman

Israel

Barry Condron

USA

Frank Conlon

USA

Stephanie Cossette

USA

Selahattin Danisman

Germany

Fiorenza De Bernardi

Italy

Katia Del Rio-Tsonis

USA

Franck Delaunay

France

Alison Dell

USA

\author{
Herman Dierick \\ USA \\ Virginia Diewert \\ Canada \\ Wolfgang Driever \\ Germany \\ Richard M. Epand \\ Canada \\ Maria-José Escribá \\ Spain
}

Alexei Evsikov

USA

Paul Fowler

UK

Tamara Franz-Odendaal

Canada

Brian Gabrielli

Australia

Gary Gaufo

USA

Burhan Gharaibeh

USA

Roman Giger

USA

Sarah Goetz

USA

Kaoru Goto

Japan

Correspondence: Philippa.Harris@biomedcentral.com

BioMed Central, 236 Gray's Inn Road, London WC1X 8HB, UK 


\begin{tabular}{|c|c|c|}
\hline Mukesh Kumar Gupta & Lingyu Li & Heiner Niemann \\
\hline India & USA & Germany \\
\hline Marnie Halpern & Ziyi Li & Hirotaka James Okano \\
\hline USA & China & Japan \\
\hline Andreas Heyland & Ching-Ling (Ellen) Lien & Katsuhiko Ono \\
\hline Canada & USA & Japan \\
\hline Andreas Hiltbrunner & Gufa Lin & Christopher Ormandy \\
\hline Germany & USA & Australia \\
\hline Seiji Hitoshi & Susana Lopes & Achim Paululat \\
\hline Japan & Portugal & Germany \\
\hline Katja Hummitzsch & Scott Lozanoff & Christopher Payne \\
\hline Australia & USA & USA \\
\hline Helen Irving-Rodgers & P Mabee & Natalie Payne \\
\hline Australia & USA & Australia \\
\hline Jr Jacobs & Paul Maddox & Francisco Pelegri \\
\hline Canada & USA & USA \\
\hline Anna Jazwinska & Fanny Mann & Roberta Pennati \\
\hline Switzerland & France & Italy \\
\hline Yasuhiko Kawakami & Julio Martin & Christian Petersen \\
\hline USA & Spain & USA \\
\hline Nobuyuki Kawashima & Maria D Martin-Bermudo & M.Dolors Piulachs \\
\hline Japan & Spain & Spain \\
\hline Wiliam Kelly & Chris Mayhew & Berenika Anna Plusa \\
\hline USA & USA & UK \\
\hline Khandan Keyomarsi & Dies Meijer & Ken Prehoda \\
\hline USA & UK & USA \\
\hline Toshikazu Kondo & Myrte Merkestein & Don Ready \\
\hline Japan & UK & USA \\
\hline Ping Kong & Anthony Mescher & Joao Relvas \\
\hline USA & USA & Portugal \\
\hline Laijun Lai & Mark Metzstein & Rene Rezsohazy \\
\hline USA & USA & Belgium \\
\hline Tilman Lamparter & Silvia Clotilde Modina & Sandra Rieger \\
\hline Germany & Italy & USA \\
\hline Pierre Leclerc & Denise Montell & Filippo Rijli \\
\hline Canada & USA & Switzerland \\
\hline Armand Leroi & Bernard Moussian & Paul Riley \\
\hline UK & Germany & UK \\
\hline Michael Levin & David Murphy & Rakel Romar \\
\hline USA & UK & Spain \\
\hline Gil Levkowitz & Christopher Navara & Siegfried Roth \\
\hline Israel & USA & Germany \\
\hline Baojie Li & Xavier Nicol & Charles Sagerstrom \\
\hline China & France & USA \\
\hline
\end{tabular}


Jean-Luc Scemama

USA

Sylvie Schneider-Maunoury

France

Norito Shibata

Japan

Fraser Sim

USA

Johan Smitz

Belgium

Josefa Steinhauer

USA

Scott Stewart

USA

Michael Stock

Germany

Padraig Strappe

Australia

Devi Suman

USA

Genlou Sun

Canada

Beat Suter

Switzerland
Tohru Suzuki

Japan

Piroska Szabo

USA

Qinghua Tao

China

Kevin Temeyer

USA

$\mathrm{X}$ Cindy Tian

USA

Simon Tuck

Sweden

Alexander Vaiserman

Ukraine

Michael Todd Valerius

USA

Srinivasan Vijayaraghavan

USA

Xin Wang

USA

Timothy Weil

UK

Gunther Wennemuth

Germany
Gary Wessel

USA

Gabrielle Wheway

UK

Gregory Wray

USA

Chun-Fang Wu

USA

Xuechun Xia

China

Ting Xie

USA

Yukiko Yamazaki

USA

Gyesoon Yoon

South Korea

Xin Yuan

USA

Vincenzo Zappavigna

Italy

Meijia Zhang

China 